Jingjing Sun, 2022

Volume 7 Issue 3, 21-40.

Received: 04 ${ }^{\text {th }}$ May 2021

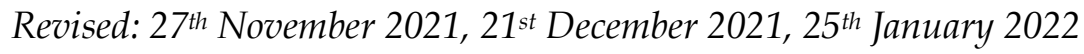

Accepted: 26 th January 2022

Date of Publication: $28^{\text {th }}$ January 2022

DOI- https://dx.doi.org/10.20319/lijhls.2022.73.2140

This paper can be cited as Jingiing, S. (2022). Influence of recuperation environment in medical institutions on chronic pain in elderly patients and analysis of protective measures LIFE: International Journal of Health and Life-Sciences, 7(3) 21-40.

This work is licensed under the CreativeCommons Attribution-Non-commercial 4.0 International License. To view a copy of this license, visit http://creativecommons.org/licenses/by-nc/4.0/ or send a letter to Creative Commons, PO Box 1866, Mountain View, CA 94042, USA

\title{
INFLUENCE OF RECUPERATION ENVIRONMENT ON CHRONIC PAIN IN ELDERLY PATIENTS
}

\author{
Jingjing Sun \\ PhD student, Research Institute of Architecture, \\ Southeast University, Nanjing, China \\ 150420200@qq.com
}

\begin{abstract}
Chronic pain is a common disease of elderly patients, and it is also a factor that has a significant impact on the physical, mental health, and quality of life of elderly patients. In terms of the existing research, most of the relevant fields focus on the diagnosis and treatment of chronic pain, and various alternative therapies have also been studied and discussed on a certain scale. This paper is more from the overall rehabilitation work of medical institutions, for patients with chronic pain, this paper analyzes the factors which influence, not only consider a patient's nursing intervention but also considers the patient's medical workplace environment, etc., for a variety of factors, affect the elderly patients with chronic pain conditions and corresponding protection measures are analyzed.
\end{abstract}




\section{Keywords}

Recuperation Environment, Elderly Patients, Chronic Pain, Medical Institutions

\section{Introduction}

Acute pain is a kind of feeling with important value, which can help the group to react quickly to prevent infringement and recover losses. Chronic pain is a common symptom of a variety of diseases, manifested as a kind of physical and psychological discomfort, is a kind of unpleasant physical and emotional experience, usually recurrent, protracted. Both acute pain and chronic pain indicate corresponding tissue damage, which has important value in clinical diagnosis. For the patients, the signal value of chronic pain is weak, and the unhappiness is more significant, resulting in a significant deterioration of the quality of life.

Elderly patients are a high incidence of chronic pain. Chronic pain significantly affects the quality of life of the elderly, and an effective recuperation environment can effectively improve and protect chronic pain, which has been widely studied.

In this study, data from 274 chronic pain patients in inpatient geriatric wards of medical institutions were collected on August 20, 2019 and October 4, 2019. Questionnaire as well as data analysis results showed that patients with common for evaluation of landscape maintenance and higher for the evaluation of equipment and facilities, in patients with chronic pain in addition to show the pain feeling, also show the anxiety and fear, insomnia, and the use of antipsychotic medications, to go out for a walk, and other common activities. The condition of equipment and facilities, especially the experience of hospital bed use $(\mathrm{P}<0.01)$, significantly affected the pain perception of patients; The special features of landscape maintenance were the sports sites $(\mathrm{P}<$ 0.01), which significantly affected the pain perception of patients; Artificial service, especially service attitude $(\mathrm{P}<0.01)$ significantly affected patients' pain perception. For the overall feeling of chronic pain, the structural equation model analysis results show that the artificial service for patients with chronic pain condition has a significant effect $(\mathrm{p}<0.01)$, and facilities for patients with chronic pain conditions affect significantly weaker $(\mathrm{p}<0.1)$, landscape maintenance for patients with chronic pain conditions influence was not significant. The effect of each subdivision index on the artificial service was generally significant and strong, and the highest coefficient was service attitude $(\mathrm{P}<0.01)$, indicating that the service attitude had a significant impact on patients' chronic pain. Based on this, it can be seen that nursing intervention activities in the recuperation 
environment of medical institutions, especially the service attitude of the staff, have a significant impact on patients' chronic pain. Therefore, in order to reduce chronic pain in patients with symptoms and effects, improve patient quality of life, the medical establishment environment needs through ascension and improve nursing intervention level, improve the quality of service methods such as the certain protective measures for patients with chronic pain, and more with the introduction of interpersonal communication activities and sports exercise program and so on ways to improve the patient's social environment, so as to reduce the patients suffering from chronic pain and improve the patient's symptoms.

\section{Literature Review}

Wang Xinjing et al. (2020) compared the effects of fire needle therapy on pain and serological characteristics of patients with lumbar disc herniation treated in a sanatorium from 2017 to 2019, and the results showed that the pain score was significantly improved by fire needle therapy. Jiang Yanfang et al. (2020) compared the effect of massage on 78 patients with shoulder, neck, low back, and leg pain treated in 2019, and the results showed that massage can effectively relieve the degree of pain. Pei Jinxue et al. (2020) tested the effect of individualized hot spring recuperation and physical recuperation methods on 86 patients with chronic low back and leg pain from 2018 to 2019, and the results showed that it can effectively reduce the pain of patients. Xu Lihua et al. (2020) compared the effect of emotional release therapy on elderly patients with chronic pain, and the results showed that emotional release therapy could improve the degree of pain, anxiety, and depression of patients. Fu Xinying et al. (2020) used 73 patients with lumbar disc herniation from 2016 to 2019 to compare the effect of lumbodorsal muscle functional exercise. The results showed that lumbodorsal muscle functional exercise can effectively improve the pain feeling of patients. Li Lei Xiaoli (2019) analyzed the effect of nursing intervention on elderly patients with chronic pain, and the results showed that nursing intervention in the treatment of chronic pain can significantly reduce the degree of pain of patients. Hira Islam Rajput et al. (2019) used a cross-sectional study to collect shoulder and neck pain data of office workers aged 22 to 41 . The results showed that the prevalence rate of work-related neck pain and shoulder pain was $72 \%$, and the symptoms increased with the increase of computer exposure time. Cao Chenguang et al. (2019) compared and analyzed the effect of yoga on the improvement of occupational low back 
pain in submarine officers and soldiers. The results showed that Yoga treatment can significantly improve the pain and improve the patient's endurance and satisfaction.

Generally speaking, this kind of adjuvant therapy and intervention generally has a certain effect on the pain improvement of patients but also shows the huge scale of patients with chronic pain.

Significantly, postoperative chronic pain has been more attention and emphasis, the corresponding research is more abundant. An Lijuan (2020) discussed the risk factors of chronic pain after open inguinal hernia repair in 666 cases from 2014 to 2018 . The results showed that BMI and age were the risk factors.

Lu Yi et al. (2019) used the data of 177 patients undergoing hip arthroplasty under combined spinal-epidural anesthesia to test the risk factors of pain after hip arthroplasty in the elderly and constructed a risk assessment model. The results showed that larger intraoperative blood loss, longer operation time, and postoperative NRS score were the risk factors. Gao Meihua (2019) analyzed the data of 91 elderly recuperations. The results showed that the past occupation and the number of chronic diseases were the risk factors of chronic pain.

In addition, some studies have done more analysis on the impact of chronic pain. Zhu Chengwei et al. (2020) designed a questionnaire to investigate the relationship between pain degree, pain impact degree, and quality of life for elderly patients and other elderly people in the community. The results showed that the negative correlation between them was statistically significant.

There are also many literature reviews in related fields. Li Xiahui et al. (2020) reviewed the health education of elderly patients with chronic pain and discussed the form, content, and evaluation system of such health education. Han Jian et al. (2020) made a literature review on the self-management of elderly patients with chronic pain and made corresponding comments and Analyses on this kind of self-management mode and chronic pain itself. Wang Jianhua et al. (2019) reviewed the research on the quality of life of elderly patients with chronic pain, and reviewed and discussed the influencing factors and measurement tools of the quality of life of elderly patients with chronic pain. Chen Xiaohui et al. (2019) reviewed the mechanism of cognitive impairment caused by postoperative pain in elderly patients. Yan Fei (2019) reviewed the literature on the management of chronic pain in the elderly, and reviewed and discussed the definition, development, evaluation, and intervention of chronic pain. The research direction of this kind of 
review is more extensive, for different types of chronic pain, especially postoperative pain is analyzed and discussed, and clinical and alternative therapy are reviewed and discussed.

\section{Experimental Methods}

The experimental methods consist of defining index selection and setting up and distributing questionnaires.

\subsection{Index Selection}

Based on the previous research results, the evaluation index system of chronic pain of patients and recuperation environment of medical institutions was established, as shown in the table below:

Table 1: Evaluation System

\begin{tabular}{|c|c|c|c|}
\hline First level indicators & $\begin{array}{l}\text { Second level } \\
\text { indicators }\end{array}$ & Third level indicators & $\begin{array}{l}\text { Index } \\
\text { code }\end{array}$ \\
\hline \multirow{14}{*}{$\begin{array}{l}\text { Recuperation } \\
\text { environment }\end{array}$} & \multirow{5}{*}{$\begin{array}{l}\text { Landscape } \\
\text { maintenance }\end{array}$} & Ornament & a11 \\
\hline & & Indoor green plants & a12 \\
\hline & & Outdoor greenery & a13 \\
\hline & & Leisure space & a14 \\
\hline & & Sports space & a15 \\
\hline & \multirow{7}{*}{$\begin{array}{l}\text { Equipment and } \\
\text { facilities }\end{array}$} & Hospital bed use experience & a21 \\
\hline & & Ward area per capita & $\mathrm{a} 22$ \\
\hline & & Bedside examination & a23 \\
\hline & & Fixed inspection equipment & a24 \\
\hline & & Rehabilitation facilities & $\mathrm{a} 25$ \\
\hline & & Safety measures & a26 \\
\hline & & Meals & a31 \\
\hline & \multirow{4}{*}{ Human services } & Wash & a32 \\
\hline & & Rehabilitation & a33 \\
\hline \multirow{14}{*}{ Chronic pain } & & Physical examination & a34 \\
\hline & & Service attitude & a35 \\
\hline & \multirow{5}{*}{ Emotion } & Fear & b11 \\
\hline & & Depressed & b12 \\
\hline & & Anxious & b13 \\
\hline & & Nervous & b14 \\
\hline & & Tired out & b15 \\
\hline & \multirow[t]{2}{*}{ Pain } & Pain feeling & b21 \\
\hline & & Insomnia & b31 \\
\hline & \multirow{4}{*}{ Performance } & Self-control & b32 \\
\hline & & Working ability & b33 \\
\hline & & Independent ability & b34 \\
\hline & & Tranquilizers & b35 \\
\hline & Everyday behavior & Clean up the room & $\mathrm{b} 41$ \\
\hline
\end{tabular}




$\begin{array}{ll}\text { Go for a walk } & \text { b42 } \\ \text { Getting along with family } & \text { b43 } \\ \text { Shopping and leisure } & \text { b44 } \\ \text { Spare time activities and } & \text { b45 } \\ \text { exercise } & \end{array}$

(Source: Conducted by Author)

As shown in the table above, the questionnaire is divided into two parts: recuperation environment assessment and chronic pain assessment, with a total of 32 indicators. Among them, the recuperation environment contains three kinds of indicators, landscape maintenance, equipment and facilities, and artificial services independently play a role in chronic pain. Chronic pain is a complex and systematic concept. All pain is composed of psychological components, which can be expressed in different aspects. Since the first mock exam of gate control theory was put forward in the 1960s, pain psychology has been well developed. Researchers have been able to consider the psychological models of pain from a macroscopic perspective, observe the psychological factors activating and regulating nociception and pain, and then turn the concept of pain into a psychophysiological behavior society complex structure. On this basis, the theory can be constructed.

\subsection{Development of a Questionnaire}

The main part of the questionnaire can be formed by transforming the above 32 indicators into items. The item selection of this questionnaire is a total of five levels of indicators of the Likert equidistance scale, that is, the results that need to be evaluated and judged by the interviewees are the indicators that the differences among different options are the same, and the expression mode of each option is unified, and the options are very poor, poor, medium, good and excellent. The form of the five-level scale is in the options, and it changes to 1-5 when the data is entered, so the direction of the evaluation index is positive.

In addition, the questionnaire should also indicate that the preparation, distribution, and data utilization of the questionnaire is only for academic purposes, and do not collect, retain or disclose the personal information of the interviewees.

\subsection{Distribution and Recovery of the Questionnaire}

The questionnaire was distributed to patients with chronic pain in medical institutions. For this reason, the questionnaire is printed with a paper questionnaire, which will be inquired and distributed in the geriatric ward of a hospital from August 20, 2020, to October 4, 2020. Only the inpatients in the hospital will be distributed. The source, purpose, and content of the questionnaire 
will be explained and explained to the patients on the spot. The questionnaire will be distributed after the patients acknowledge, will fill in the questionnaire, and fully understand the content of the questionnaire After completion, it will be recovered on site.

A total of 300 questionnaires were distributed and 286 were collected on-site, with a recovery rate of $95.3 \%$. Based on the collected questionnaires, 274 valid questionnaires were obtained by eliminating blank questionnaires, unfinished questionnaires, and defaced questionnaires. The effective rate of the questionnaire was $95.8 \%$, and the overall effective rate of questionnaire recovery was $91.3 \%$, which was relatively high. Based on this, the information of the questionnaire is input, and the input data is used for further processing and analysis.

\section{Experimental Results}

The experimental results are tested through reliability and validity analysis, and then analyzed through descriptive statistics. Then structural equation model analysis is carried out based on the data generated via Hierarchical Regression.

\subsection{Reliability and Validity Analysis}

The results are as follows:

Table 2: Reliability Analysis

\begin{tabular}{llll}
\hline Number of items & & Sample size & \multicolumn{3}{c}{ Cronbach $\alpha$ coefficient } & 0.757 \\
\hline
\end{tabular}

(Source: conducted by author)

As shown in the above table, the Cronbach $\alpha$ coefficient of the questionnaire data reaches 0.757, which is at a high level, indicating that the reliability of the questionnaire data is good. Then, the validity analysis was carried out, and the results were as follows:

Table 3: Validity Analysis

\begin{tabular}{|c|c|c|c|c|c|c|c|c|c|}
\hline Factor load factor & $\begin{array}{l}\text { Fact } \\
\text { or } 1\end{array}$ & $\begin{array}{l}\text { Fact } \\
\text { or } 2\end{array}$ & $\begin{array}{l}\text { Fact } \\
\text { or } 3\end{array}$ & $\begin{array}{l}\text { Fact } \\
\text { or } 4\end{array}$ & $\begin{array}{l}\text { Fact } \\
\text { or } 5\end{array}$ & $\begin{array}{l}\text { Fact } \\
\text { or } 6\end{array}$ & $\begin{array}{l}\text { Fact } \\
\text { or } 7\end{array}$ & $\begin{array}{l}\text { Fact } \\
\text { or } 8\end{array}$ & $\begin{array}{l}\text { Commu } \\
\text { nality }\end{array}$ \\
\hline $\mathrm{a} 22$ & $\begin{array}{r}- \\
0.14 \\
4\end{array}$ & $\begin{array}{r}- \\
0.02 \\
5\end{array}$ & $\begin{array}{r}0.61 \\
2\end{array}$ & $\begin{array}{r}- \\
0.14 \\
9\end{array}$ & $\begin{array}{r}0.29 \\
3\end{array}$ & $\begin{array}{r}0.06 \\
2\end{array}$ & $\begin{array}{r}0.03 \\
8\end{array}$ & $\begin{array}{r}- \\
0.27 \\
9\end{array}$ & 0.588 \\
\hline $\mathrm{a} 21$ & $\begin{array}{r}0.37 \\
9\end{array}$ & $\begin{array}{r}- \\
0.08 \\
4\end{array}$ & $\begin{array}{r}0.64 \\
5\end{array}$ & $\begin{array}{r}0.16 \\
9\end{array}$ & -0.18 & -0.01 & $\begin{array}{r}0.12 \\
8\end{array}$ & $\begin{array}{r}0.15 \\
6\end{array}$ & 0.668 \\
\hline
\end{tabular}




\begin{tabular}{|c|c|c|c|c|c|c|c|c|c|}
\hline a23 & $\begin{array}{r}0.08 \\
2\end{array}$ & $\begin{array}{r}0.00 \\
8\end{array}$ & 0.76 & 0.08 & $\begin{array}{r}- \\
0.04 \\
8\end{array}$ & $\begin{array}{r}- \\
0.03 \\
5\end{array}$ & $\begin{array}{r}- \\
0.01 \\
7\end{array}$ & $\begin{array}{r}0.05 \\
5\end{array}$ & 0.598 \\
\hline a24 & $\begin{array}{r}0.28 \\
4\end{array}$ & -0.03 & $\begin{array}{r}0.70 \\
2\end{array}$ & $\begin{array}{r}0.06 \\
9\end{array}$ & $\begin{array}{r}- \\
0.21 \\
9\end{array}$ & $\begin{array}{r}0.05 \\
7\end{array}$ & $\begin{array}{r}0.08 \\
2\end{array}$ & $\begin{array}{r}0.00 \\
5\end{array}$ & 0.637 \\
\hline $\mathrm{a} 25$ & $\begin{array}{r}0.32 \\
3\end{array}$ & $\begin{array}{r}0.02 \\
7\end{array}$ & $\begin{array}{r}0.52 \\
9\end{array}$ & $\begin{array}{r}0.07 \\
2\end{array}$ & $\begin{array}{r}- \\
0.12 \\
7\end{array}$ & 0.05 & 0.19 & $\begin{array}{r}0.22 \\
2\end{array}$ & 0.494 \\
\hline a26 & $\begin{array}{r}0.17 \\
5\end{array}$ & $\begin{array}{r}- \\
0.12 \\
9\end{array}$ & $\begin{array}{r}0.13 \\
4\end{array}$ & $\begin{array}{r}0.11 \\
2\end{array}$ & $\begin{array}{r}- \\
0.12 \\
9\end{array}$ & $\begin{array}{r}0.07 \\
2\end{array}$ & $\begin{array}{r}0.74 \\
9\end{array}$ & $\begin{array}{r}0.03 \\
9\end{array}$ & 0.661 \\
\hline a12 & $\begin{array}{r}0.28 \\
2\end{array}$ & $\begin{array}{r}0.02 \\
6\end{array}$ & 0.05 & $\begin{array}{r}0.21 \\
2\end{array}$ & $\begin{array}{r}- \\
0.10 \\
7\end{array}$ & $\begin{array}{r}0.00 \\
4\end{array}$ & $\begin{array}{r}0.71 \\
7\end{array}$ & $\begin{array}{r}0.19 \\
7\end{array}$ & 0.691 \\
\hline a11 & $\begin{array}{r}0.21 \\
4\end{array}$ & $\begin{array}{r}- \\
0.03 \\
7\end{array}$ & 0.06 & $\begin{array}{r}0.81 \\
4\end{array}$ & $\begin{array}{r}- \\
0.12 \\
7\end{array}$ & $\begin{array}{r}- \\
0.03 \\
3\end{array}$ & $\begin{array}{r}0.16 \\
4\end{array}$ & $\begin{array}{r}0.08 \\
4\end{array}$ & 0.765 \\
\hline a13 & $\begin{array}{r}0.21 \\
7\end{array}$ & $\begin{array}{r}0.07 \\
1\end{array}$ & $\begin{array}{r}0.07 \\
6\end{array}$ & $\begin{array}{r}0.56 \\
4\end{array}$ & $\begin{array}{r}0.04 \\
1\end{array}$ & $\begin{array}{r}- \\
0.01 \\
6\end{array}$ & $\begin{array}{r}0.15 \\
5\end{array}$ & $\begin{array}{r}0.05 \\
2\end{array}$ & 0.405 \\
\hline a14 & $\begin{array}{r}0.20 \\
5\end{array}$ & $\begin{array}{r}- \\
0.01 \\
9\end{array}$ & $\begin{array}{r}0.04 \\
5\end{array}$ & $\begin{array}{r}0.86 \\
7\end{array}$ & $\begin{array}{r}- \\
0.05 \\
9\end{array}$ & 0.05 & $\begin{array}{r}- \\
0.00 \\
5\end{array}$ & $\begin{array}{r}0.07 \\
1\end{array}$ & 0.808 \\
\hline a15 & $\begin{array}{r}0.81 \\
6\end{array}$ & $\begin{array}{r}0.01 \\
3\end{array}$ & $\begin{array}{r}0.04 \\
3\end{array}$ & $\begin{array}{r}0.18 \\
1\end{array}$ & $\begin{array}{r}- \\
0.07 \\
1\end{array}$ & $\begin{array}{r}0.07 \\
1\end{array}$ & $\begin{array}{r}0.04 \\
9\end{array}$ & $\begin{array}{r}- \\
0.04 \\
9\end{array}$ & 0.715 \\
\hline a32 & $\begin{array}{r}0.85 \\
2\end{array}$ & $\begin{array}{r}0.03 \\
7\end{array}$ & 0.04 & $\begin{array}{r}0.16 \\
2\end{array}$ & $\begin{array}{r}- \\
0.15 \\
9\end{array}$ & 0.06 & $\begin{array}{r}0.02 \\
8\end{array}$ & $\begin{array}{r}0.04 \\
2\end{array}$ & 0.787 \\
\hline a31 & $\begin{array}{r}0.87 \\
5\end{array}$ & $\begin{array}{r}- \\
0.04 \\
3\end{array}$ & $\begin{array}{r}0.04 \\
2\end{array}$ & $\begin{array}{r}0.10 \\
8\end{array}$ & $\begin{array}{r}- \\
0.07 \\
1\end{array}$ & $\begin{array}{r}- \\
0.03 \\
7\end{array}$ & $\begin{array}{r}0.09 \\
3\end{array}$ & $\begin{array}{r}- \\
0.09 \\
3\end{array}$ & 0.804 \\
\hline a33 & 0.82 & $\begin{array}{r}- \\
0.09 \\
2\end{array}$ & $\begin{array}{r}0.15 \\
6\end{array}$ & $\begin{array}{r}0.15 \\
9\end{array}$ & $\begin{array}{r}- \\
0.04 \\
7\end{array}$ & $\begin{array}{r}0.10 \\
4\end{array}$ & $\begin{array}{r}0.05 \\
5\end{array}$ & $\begin{array}{r}- \\
0.03 \\
5\end{array}$ & 0.748 \\
\hline a34 & $\begin{array}{r}0.59 \\
1\end{array}$ & $\begin{array}{r}- \\
0.01 \\
7\end{array}$ & $\begin{array}{r}0.18 \\
6\end{array}$ & 0.16 & $\begin{array}{r}- \\
0.04 \\
7\end{array}$ & $\begin{array}{r}- \\
0.07 \\
3\end{array}$ & $\begin{array}{r}0.36 \\
2\end{array}$ & $\begin{array}{r}0.19 \\
7\end{array}$ & 0.588 \\
\hline a35 & $\begin{array}{r}0.60 \\
5\end{array}$ & $\begin{array}{r}0.00 \\
6\end{array}$ & $\begin{array}{r}0.23 \\
4\end{array}$ & $\begin{array}{r}0.13 \\
3\end{array}$ & $\begin{array}{r}- \\
0.19 \\
9\end{array}$ & $\begin{array}{r}- \\
0.04 \\
6\end{array}$ & $\begin{array}{r}0.30 \\
4\end{array}$ & $\begin{array}{r}0.36 \\
5\end{array}$ & 0.705 \\
\hline b2 & $\begin{array}{r}- \\
0.53 \\
5\end{array}$ & $\begin{array}{r}0.01 \\
5\end{array}$ & $\begin{array}{r}- \\
0.21 \\
7\end{array}$ & $\begin{array}{r}- \\
0.09 \\
2\end{array}$ & $\begin{array}{r}0.28 \\
5\end{array}$ & $\begin{array}{r}- \\
0.01 \\
6\end{array}$ & -0.04 & $\begin{array}{r}- \\
0.31 \\
4\end{array}$ & 0.523 \\
\hline
\end{tabular}




\begin{tabular}{|c|c|c|c|c|c|c|c|c|c|}
\hline b12 & $\begin{array}{r}0.58 \\
1\end{array}$ & $\begin{array}{r}- \\
0.06 \\
3\end{array}$ & $\begin{array}{r}0.23 \\
2\end{array}$ & $\begin{array}{r}0.10 \\
6\end{array}$ & $\begin{array}{r}- \\
0.25 \\
2\end{array}$ & $\begin{array}{r}- \\
0.03 \\
2\end{array}$ & $\begin{array}{r}0.21 \\
8\end{array}$ & $\begin{array}{r}0.36 \\
7\end{array}$ & 0.654 \\
\hline b11 & $\begin{array}{r}- \\
0.15 \\
5\end{array}$ & $\begin{array}{r}- \\
0.01 \\
7\end{array}$ & $\begin{array}{r}- \\
0.10 \\
8\end{array}$ & $\begin{array}{r}- \\
0.02 \\
9\end{array}$ & 0.68 & $\begin{array}{r}- \\
0.11 \\
3\end{array}$ & $\begin{array}{r}- \\
0.19 \\
3\end{array}$ & 0.06 & 0.552 \\
\hline b13 & $\begin{array}{r}- \\
0.10 \\
4\end{array}$ & $\begin{array}{r}0.00 \\
1\end{array}$ & $\begin{array}{r}0.00 \\
1\end{array}$ & $\begin{array}{r}- \\
0.02 \\
5\end{array}$ & $\begin{array}{r}0.78 \\
7\end{array}$ & $\begin{array}{r}- \\
0.02 \\
7\end{array}$ & $\begin{array}{r}- \\
0.08 \\
5\end{array}$ & $\begin{array}{r}0.14 \\
1\end{array}$ & 0.659 \\
\hline b14 & -0.33 & $\begin{array}{r}0.02 \\
3\end{array}$ & $\begin{array}{r}- \\
0.15 \\
2\end{array}$ & $\begin{array}{r}- \\
0.07 \\
7\end{array}$ & $\begin{array}{r}0.63 \\
3\end{array}$ & $\begin{array}{r}- \\
0.04 \\
9\end{array}$ & $\begin{array}{r}0.06 \\
3\end{array}$ & $\begin{array}{r}- \\
0.17 \\
1\end{array}$ & 0.575 \\
\hline b15 & $\begin{array}{r}0.60 \\
5\end{array}$ & $\begin{array}{r}0.02 \\
2\end{array}$ & $\begin{array}{r}0.19 \\
8\end{array}$ & $\begin{array}{r}0.07 \\
5\end{array}$ & $\begin{array}{r}- \\
0.19 \\
1\end{array}$ & -0.04 & $\begin{array}{r}0.20 \\
3\end{array}$ & $\begin{array}{r}0.46 \\
7\end{array}$ & 0.708 \\
\hline b32 & $\begin{array}{r}0.08 \\
8\end{array}$ & $\begin{array}{r}- \\
0.07 \\
7\end{array}$ & $\begin{array}{r}0.01 \\
1\end{array}$ & $\begin{array}{r}0.14 \\
4\end{array}$ & $\begin{array}{r}0.20 \\
8\end{array}$ & $\begin{array}{r}0.05 \\
7\end{array}$ & 0.15 & $\begin{array}{r}0.67 \\
6\end{array}$ & 0.56 \\
\hline b31 & $\begin{array}{r}- \\
0.06 \\
5\end{array}$ & $\begin{array}{r}0.59 \\
4\end{array}$ & 0.11 & 0.03 & $\begin{array}{r}0.07 \\
7\end{array}$ & $\begin{array}{r}- \\
0.17 \\
3\end{array}$ & $\begin{array}{r}0.15 \\
9\end{array}$ & $\begin{array}{r}- \\
0.23 \\
8\end{array}$ & 0.488 \\
\hline b33 & $\begin{array}{r}0.00 \\
6\end{array}$ & $\begin{array}{r}0.76 \\
3\end{array}$ & $\begin{array}{r}- \\
0.02 \\
8\end{array}$ & $\begin{array}{r}- \\
0.04 \\
1\end{array}$ & -0.04 & $\begin{array}{r}0.22 \\
4\end{array}$ & $\begin{array}{r}- \\
0.12 \\
1\end{array}$ & $\begin{array}{r}0.09 \\
9\end{array}$ & 0.661 \\
\hline b34 & $\begin{array}{r}- \\
0.03 \\
6\end{array}$ & $\begin{array}{r}0.80 \\
2\end{array}$ & $\begin{array}{r}0.00 \\
8\end{array}$ & $\begin{array}{r}0.07 \\
2\end{array}$ & 0.02 & $\begin{array}{r}0.03 \\
4\end{array}$ & $\begin{array}{r}0.00 \\
6\end{array}$ & $\begin{array}{r}- \\
0.05 \\
2\end{array}$ & 0.654 \\
\hline b35 & $\begin{array}{r}- \\
0.01 \\
4\end{array}$ & $\begin{array}{r}0.86 \\
4\end{array}$ & $\begin{array}{r}- \\
0.05 \\
4\end{array}$ & $\begin{array}{r}- \\
0.02 \\
1\end{array}$ & $\begin{array}{r}- \\
0.05 \\
8\end{array}$ & $\begin{array}{r}0.00 \\
4\end{array}$ & $\begin{array}{r}- \\
0.06 \\
3\end{array}$ & $\begin{array}{r}- \\
0.04 \\
6\end{array}$ & 0.76 \\
\hline b42 & $\begin{array}{r}- \\
0.00 \\
1\end{array}$ & $\begin{array}{r}0.71 \\
2\end{array}$ & $\begin{array}{r}- \\
0.08 \\
8\end{array}$ & $\begin{array}{r}- \\
0.01 \\
5\end{array}$ & $\begin{array}{r}0.01 \\
8\end{array}$ & $\begin{array}{r}0.16 \\
8\end{array}$ & $\begin{array}{r}- \\
0.07 \\
8\end{array}$ & $\begin{array}{r}0.08 \\
7\end{array}$ & 0.557 \\
\hline b41 & $\begin{array}{r}0.04 \\
5\end{array}$ & $\begin{array}{r}0.40 \\
4\end{array}$ & $\begin{array}{r}0.04 \\
5\end{array}$ & $\begin{array}{r}- \\
0.03 \\
3\end{array}$ & $\begin{array}{r}- \\
0.00 \\
3\end{array}$ & $\begin{array}{r}0.56 \\
3\end{array}$ & $\begin{array}{r}0.17 \\
7\end{array}$ & $\begin{array}{r}- \\
0.07 \\
4\end{array}$ & 0.522 \\
\hline b43 & $\begin{array}{r}0.06 \\
8\end{array}$ & $\begin{array}{r}0.20 \\
1\end{array}$ & $\begin{array}{r}0.12 \\
3\end{array}$ & $\begin{array}{r}0.02 \\
7\end{array}$ & $\begin{array}{r}- \\
0.02 \\
2\end{array}$ & $\begin{array}{r}0.71 \\
5\end{array}$ & $\begin{array}{r}0.16 \\
8\end{array}$ & $\begin{array}{r}- \\
0.16 \\
5\end{array}$ & 0.629 \\
\hline b44 & $\begin{array}{r}- \\
0.00 \\
4\end{array}$ & $\begin{array}{r}- \\
0.04 \\
4\end{array}$ & $\begin{array}{r}0.08 \\
4\end{array}$ & $\begin{array}{r}- \\
0.08 \\
3\end{array}$ & $\begin{array}{r}- \\
0.08 \\
4\end{array}$ & $\begin{array}{r}0.68 \\
8\end{array}$ & $\begin{array}{r}- \\
0.14 \\
4\end{array}$ & 0.25 & 0.58 \\
\hline b45 & $\begin{array}{r}- \\
0.00 \\
4\end{array}$ & 0 & -0.1 & $\begin{array}{r}0.05 \\
2\end{array}$ & $\begin{array}{r}- \\
0.04 \\
7\end{array}$ & $\begin{array}{r}0.58 \\
1\end{array}$ & $\begin{array}{r}- \\
0.03 \\
2\end{array}$ & $\begin{array}{r}0.01 \\
8\end{array}$ & 0.354 \\
\hline
\end{tabular}




\begin{tabular}{|c|c|c|c|c|c|c|c|c|c|}
\hline $\begin{array}{l}\text { Eigenvalue } \\
\text { (Unrotated) }\end{array}$ & $\begin{array}{r}8.15 \\
1\end{array}$ & $\begin{array}{r}3.30 \\
5\end{array}$ & $\begin{array}{r}1.93 \\
1\end{array}$ & 1.7 & $\begin{array}{r}1.49 \\
4\end{array}$ & 1.34 & $\begin{array}{r}1.15 \\
6\end{array}$ & 1.02 & - \\
\hline$\%$ of Variance & 0.25 & 0.10 & 0.06 & 0.05 & 0.04 & 0.04 & 0.03 & 0.03 & - \\
\hline (Unrotated) & 471 & 328 & 035 & 313 & 668 & 188 & 612 & 189 & - \\
\hline Cumulative $\%$ of & 0.25 & 0.35 & 0.41 & 0.47 & 0.51 & 0.56 & 0.59 & 0.62 & \\
\hline Variance(Unrotated) & 471 & 799 & 834 & 147 & 815 & 004 & 615 & 804 & - \\
\hline Eigenvalue(Rotated) & $\begin{array}{r}5.29 \\
8\end{array}$ & $\begin{array}{r}3.09 \\
5\end{array}$ & $\begin{array}{r}2.52 \\
5\end{array}$ & $\begin{array}{r}2.07 \\
1\end{array}$ & $\begin{array}{r}2.04 \\
2\end{array}$ & $\begin{array}{r}1.81 \\
9\end{array}$ & $\begin{array}{r}1.71 \\
6\end{array}$ & $\begin{array}{r}1.53 \\
1\end{array}$ & - \\
\hline $\begin{array}{l}\% \text { of } \\
\text { Variance(Rotated) }\end{array}$ & $\begin{array}{r}0.16 \\
558\end{array}$ & $\begin{array}{r}0.09 \\
671\end{array}$ & $\begin{array}{r}0.07 \\
891\end{array}$ & $\begin{array}{r}0.06 \\
472\end{array}$ & $\begin{array}{r}0.06 \\
38\end{array}$ & $\begin{array}{r}0.05 \\
684\end{array}$ & $\begin{array}{r}0.05 \\
364\end{array}$ & $\begin{array}{r}0.04 \\
786\end{array}$ & \\
\hline $\begin{array}{l}\text { Cumulative } \% \text { of } \\
\text { Variance(Rotated) }\end{array}$ & $\begin{array}{r}0.16 \\
558\end{array}$ & $\begin{array}{r}0.26 \\
229\end{array}$ & $\begin{array}{r}0.34 \\
119\end{array}$ & $\begin{array}{r}0.40 \\
591\end{array}$ & $\begin{array}{r}0.46 \\
971\end{array}$ & $\begin{array}{r}0.52 \\
654\end{array}$ & $\begin{array}{r}0.58 \\
018\end{array}$ & $\begin{array}{r}0.62 \\
804\end{array}$ & 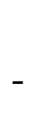 \\
\hline KMO value & & \multicolumn{7}{|c|}{0.864} & - \\
\hline $\begin{array}{l}\text { Bartlett's test of } \\
\text { sphericity }\end{array}$ & & \multicolumn{7}{|c|}{3767.184} & - \\
\hline df & & \multicolumn{7}{|c|}{496} & - \\
\hline p-value . & & \multicolumn{7}{|c|}{0} & - \\
\hline
\end{tabular}

(Source: conducted by author)

The results of validity analysis are shown in the table above, in which the kmo value reaches 0.864 , which is at an excellent level, and the validity test is passed. But in each index, the common value of B45 is too low, so the model is excluded.

\subsection{Descriptive Statistics}

Based on reliability analysis and validity analysis of the questionnaire data, descriptive statistics are carried out for the sample data to understand the overall characteristics of the data. The results are shown in the following table:

Table 4: Descriptive statistics

\begin{tabular}{lllllll}
\hline $\begin{array}{l}\text { Nam } \\
\text { e }\end{array}$ & $\begin{array}{l}\text { Sample } \\
\text { size }\end{array}$ & $\begin{array}{l}\text { Minimum } \\
\text { value }\end{array}$ & $\begin{array}{l}\text { Maximum } \\
\text { value }\end{array}$ & $\begin{array}{l}\text { Average } \\
\text { value }\end{array}$ & $\begin{array}{l}\text { Standard } \\
\text { deviation }\end{array}$ & $\begin{array}{l}\text { Median } \\
\cdot\end{array}$ \\
\hline a11 & 274 & 1 & 5 & 2.307 & 0.906 & 2 \\
a12 & 274 & 1 & 5 & 2.449 & 1.102 & 2 \\
a13 & 274 & 1 & 5 & 2.292 & 0.862 & 2 \\
a14 & 274 & 1 & 5 & 2.215 & 0.747 & 2 \\
a15 & 274 & 1 & 5 & 2.573 & 1.333 & 2 \\
a21 & 274 & 1 & 5 & 3.08 & 1.402 & 3 \\
a22 & 274 & 1 & 5 & 3.314 & 1.341 & 4 \\
a23 & 274 & 1 & 5 & 3.015 & 1.318 & 3 \\
a24 & 274 & 1 & 5 & 3.285 & 1.298 & 3.5 \\
a25 & 274 & 1 & 5 & 3.281 & 1.308 & 3 \\
a26 & 274 & 1 & 5 & 2.785 & 1.367 & 3 \\
a31 & 274 & 1 & 5 & 2.58 & 1.373 & 2 \\
\hline
\end{tabular}




\begin{tabular}{lllllll}
\hline a32 & 274 & 1 & 5 & 2.624 & 1.329 & 2 \\
a33 & 274 & 1 & 5 & 2.697 & 1.414 & 2 \\
a34 & 274 & 1 & 5 & 2.536 & 1.246 & 2 \\
a35 & 274 & 1 & 5 & 2.745 & 1.318 & 2 \\
b11 & 274 & 1 & 5 & 3.201 & 1.406 & 4 \\
b12 & 274 & 1 & 5 & 2.814 & 1.243 & 3 \\
b13 & 274 & 1 & 5 & 3.383 & 1.282 & 4 \\
b14 & 274 & 1 & 5 & 2.974 & 1.294 & 3 \\
b15 & 274 & 1 & 5 & 2.777 & 1.314 & 3 \\
b2 & 274 & 1 & 5 & 3.65 & 1.284 & 4 \\
b31 & 274 & 1 & 5 & 3.263 & 1.336 & 3 \\
b32 & 274 & 1 & 5 & 2.909 & 1.165 & 3 \\
b33 & 274 & 1 & 5 & 2.88 & 1.365 & 3 \\
b34 & 274 & 1 & 5 & 2.839 & 1.336 & 3 \\
b35 & 274 & 1 & 5 & 3.23 & 1.31 & 3.5 \\
b41 & 274 & 1 & 5 & 2.62 & 1.253 & 2 \\
b42 & 274 & 1 & 5 & 3.204 & 1.285 & 3 \\
b43 & 274 & 1 & 5 & 2.285 & 0.917 & 2 \\
b44 & 274 & 1 & 5 & 2.19 & 0.685 & 2 \\
b45 & 274 & 1 & 5 & 2.204 & 0.813 & 2 \\
\hline
\end{tabular}

(Source: conducted by author)

As shown in the table above, there are great differences between the average and standard deviation of different indicators.

The evaluation results of the indoor landscape are generally low, and the average value and standard deviation are generally low, which shows that the power of landscape maintenance in medical institutions is relatively insufficient, so that patients have a relatively unified poor evaluation. The evaluation of equipment and facilities is significantly higher, and the standard deviation is also higher, which shows that the equipment and facilities in medical institutions are relatively sound and in good condition, especially the rehabilitation facilities and fixed facilities, which shows that both of them have been better allocated in medical institutions. The higher level of standard deviation indicates that there may be some differences in the actual configuration density and usage of the equipment. The average value of manual service is at the medium level, and the standard deviation is further improved, which shows that different patients have a different evaluation of manual service, especially the standard deviation of rehabilitation activities is significantly higher, which shows that the auxiliary work of rehabilitation activities has great differences according to the specific staffing. Summarizing landscape maintenance, equipment, and facilities, and human services, the performance of equipment and facilities in the diagnosis 
and treatment environment of medical institutions is significantly better, while the landscape maintenance is significantly worse.

The patient's pain emotion performance is relatively poor, especially the anxiety and fear emotion performance is prominent, the standard deviation is high; the patient's pain feeling performance is also significantly poor, the patient and the general report significant pain feeling; the patient's specific performance is relatively poor, the patient often lose sleep and need to use diazepam drugs to help sleep; the patient's daily behavior is better, especially the patients Shopping and leisure activities can be carried out normally, and the standard deviation is very low, which shows that the daily activities of patients can still be guaranteed, but it is difficult to complete common simple activities such as walking, which shows that loneliness plays an auxiliary role in the development of chronic pain.

\subsection{Hierarchical Regression}

The results of hierarchical regression with B2 pain feeling as the dependent variable are as follows:

Table 5: Hierarchical regression analysis results

\begin{tabular}{|c|c|c|c|c|c|c|c|c|c|c|c|c|}
\hline & \multicolumn{4}{|c|}{ Layer 1} & \multicolumn{4}{|c|}{ Layer 2} & \multicolumn{4}{|c|}{ Layer 3} \\
\hline & B & $\begin{array}{l}\text { Standar } \\
\text { d error }\end{array}$ & $\mathrm{t}$ & $\mathrm{p}$ & B & $\begin{array}{l}\text { Standar } \\
\text { d error }\end{array}$ & $\mathrm{t}$ & $\mathrm{p}$ & B & $\begin{array}{l}\text { Standar } \\
\text { d error }\end{array}$ & $\mathrm{t}$ & $\mathrm{p}$ \\
\hline $\begin{array}{l}\text { Cons } \\
\text { tant }\end{array}$ & $\begin{array}{l}5.112 \\
* * *\end{array}$ & 0.268 & $\begin{array}{l}19 . \\
039\end{array}$ & 0 & & 0.331 & $\begin{array}{l}17 . \\
32\end{array}$ & 0 & $\begin{array}{l}5.708 \\
* * *\end{array}$ & 0.315 & $\begin{array}{l}18 . \\
107\end{array}$ & 0 \\
\hline a22 & 0.08 & 0.054 & $\begin{array}{l}1.4 \\
85\end{array}$ & $\begin{array}{l}0.1 \\
39\end{array}$ & 0.022 & 0.053 & $\begin{array}{l}0.4 \\
16\end{array}$ & $\begin{array}{l}0.6 \\
78\end{array}$ & $\begin{array}{l}- \\
0.004\end{array}$ & 0.051 & $\begin{array}{l}0.0 \\
75\end{array}$ & $\begin{array}{l}0.9 \\
41\end{array}$ \\
\hline a 21 & $\begin{array}{l}- \\
0.292 \\
* * *\end{array}$ & 0.066 & $\begin{array}{l}- \\
4.4 \\
09\end{array}$ & 0 & $\begin{array}{l}- \\
0.230 \\
* * *\end{array}$ & 0.065 & $\begin{array}{l}- \\
3.5 \\
17\end{array}$ & $\begin{array}{l}0.0 \\
01\end{array}$ & $\begin{array}{l}- \\
0.140 \\
* *\end{array}$ & 0.065 & $\begin{array}{l}2.1 \\
6\end{array}$ & $\begin{array}{l}0.0 \\
32\end{array}$ \\
\hline a23 & 0.084 & 0.063 & $\begin{array}{l}1.3 \\
46\end{array}$ & $\begin{array}{l}0.1 \\
79\end{array}$ & 0.083 & 0.06 & $\begin{array}{l}1.3 \\
73\end{array}$ & $\begin{array}{l}0.1 \\
71\end{array}$ & $\begin{array}{l}0.097 \\
*\end{array}$ & 0.057 & $\begin{array}{l}1.7 \\
08\end{array}$ & $\begin{array}{l}0.0 \\
89\end{array}$ \\
\hline a24 & $\begin{array}{l}- \\
0.082\end{array}$ & 0.072 & $\begin{array}{l}- \\
1.1 \\
37\end{array}$ & $\begin{array}{l}0.2 \\
56\end{array}$ & $\overline{-} .039$ & 0.07 & $\begin{array}{l}- \\
0.5 \\
55\end{array}$ & $\begin{array}{l}0.5 \\
79\end{array}$ & $\overline{-} .039$ & 0.067 & $\begin{array}{l}- \\
0.5 \\
91\end{array}$ & $\begin{array}{l}0.5 \\
55\end{array}$ \\
\hline a25 & $\begin{array}{l}- \\
0.201 \\
* * *\end{array}$ & 0.065 & $\begin{array}{l}- \\
3.0 \\
91\end{array}$ & $\begin{array}{l}0.0 \\
02\end{array}$ & $\begin{array}{l}- \\
0.155 \\
* *\end{array}$ & 0.063 & $\begin{array}{l}- \\
2.4 \\
74\end{array}$ & $\begin{array}{l}0.0 \\
14\end{array}$ & -0.07 & 0.061 & $\begin{array}{l}- \\
1.1 \\
48\end{array}$ & $\begin{array}{l}0.2 \\
52\end{array}$ \\
\hline a26 & 0.055 & 0.054 & $\begin{array}{l}- \\
1.0 \\
36\end{array}$ & $\begin{array}{l}0.3 \\
01\end{array}$ & 0.018 & 0.056 & $\begin{array}{l}0.3 \\
18\end{array}$ & $\begin{array}{l}0.7 \\
51\end{array}$ & 0.002 & 0.053 & $\begin{array}{l}0.0 \\
35\end{array}$ & $\begin{array}{l}0.9 \\
72\end{array}$ \\
\hline
\end{tabular}




\begin{tabular}{|c|c|c|c|c|c|c|c|c|c|}
\hline a12 & & $\begin{array}{l}- \\
0.059\end{array}$ & 0.072 & $\begin{array}{l}- \\
0.8 \\
15\end{array}$ & $\begin{array}{l}0.4 \\
16\end{array}$ & 0.057 & 0.072 & $\begin{array}{l}0.7 \\
96\end{array}$ & $\begin{array}{l}0.4 \\
27\end{array}$ \\
\hline a11 & & $\begin{array}{l}- \\
0.099\end{array}$ & 0.107 & $\begin{array}{l}- \\
0.9 \\
24\end{array}$ & $\begin{array}{l}0.3 \\
56\end{array}$ & $\begin{array}{l}- \\
0.052\end{array}$ & 0.102 & $\begin{array}{l}- \\
0.5 \\
05\end{array}$ & $\begin{array}{l}0.6 \\
14\end{array}$ \\
\hline a13 & & 0.004 & 0.084 & $\begin{array}{l}0.0 \\
48\end{array}$ & $\begin{array}{l}0.9 \\
61\end{array}$ & 0.011 & 0.08 & $\begin{array}{l}0.1 \\
38\end{array}$ & $\begin{array}{l}0.8 \\
9\end{array}$ \\
\hline a14 & & $-\overline{0}$ & 0.127 & $\begin{array}{l}- \\
0.2 \\
01\end{array}$ & $\begin{array}{l}0.8 \\
41\end{array}$ & -0.01 & 0.121 & $\begin{array}{l}- \\
0.0 \\
82\end{array}$ & $\begin{array}{l}0.9 \\
35\end{array}$ \\
\hline a15 & & $\begin{array}{l}- \\
0.268 \\
* * *\end{array}$ & 0.057 & $\begin{array}{l}- \\
4.7 \\
17\end{array}$ & 0 & $\begin{array}{l}- \\
0.148 \\
* *\end{array}$ & 0.073 & $\begin{array}{l}- \\
2.0 \\
38\end{array}$ & $\begin{array}{l}0.0 \\
43\end{array}$ \\
\hline a32 & & & & & & -0.03 & 0.083 & $\begin{array}{l}- \\
0.3 \\
56\end{array}$ & $\begin{array}{l}0.7 \\
22\end{array}$ \\
\hline a31 & & & & & & 0.091 & 0.08 & $\begin{array}{l}1.1 \\
27\end{array}$ & $\begin{array}{l}0.2 \\
61\end{array}$ \\
\hline a33 & & & & & & $\begin{array}{l}- \\
0.161 \\
* *\end{array}$ & 0.071 & $\begin{array}{l}- \\
2.2 \\
78\end{array}$ & $\begin{array}{l}0.0 \\
24\end{array}$ \\
\hline a34 & & & & & & 0.026 & 0.075 & $\begin{array}{l}0.3 \\
51\end{array}$ & $\begin{array}{l}0.7 \\
26\end{array}$ \\
\hline a35 & & & & & & $\begin{array}{l}- \\
0.359 \\
* * *\end{array}$ & 0.076 & $\begin{array}{l}- \\
4.7 \\
43\end{array}$ & 0 \\
\hline $\mathrm{R}^{2}$ & 0.252 & 0.332 & & & & 0.418 & & & \\
\hline $\begin{array}{l}\text { Adju } \\
\text { sted } \\
\mathrm{R}^{\mathbf{2}}\end{array}$ & 0.235 & 0.304 & & & & 0.382 & & & \\
\hline $\begin{array}{l}\mathrm{F} \\
\text { value }\end{array}$ & $\begin{array}{l}F(6,267)=14.983 \\
p=0.000\end{array}$ & \multicolumn{4}{|c|}{$\begin{array}{l}F(11,262)=11.823 \\
p=0.000\end{array}$} & \multicolumn{4}{|c|}{$\begin{array}{l}F(16,257)=11.538 \\
p=0.000\end{array}$} \\
\hline$\triangle R^{2}$ & 0.252 & \multicolumn{4}{|c|}{0.08} & \multicolumn{4}{|l|}{0.086} \\
\hline $\begin{array}{l}\triangle \mathrm{F} \\
\text { value }\end{array}$ & $\begin{array}{l}F(6,267)=14.983, \\
p=0.000\end{array}$ & \multicolumn{4}{|c|}{$F(5,262)=6.260, p=0.000$} & \multicolumn{4}{|c|}{$F(5,257)=7.623, p=0.000$} \\
\hline
\end{tabular}

Dependent variable: $\mathrm{b} 2$

$* \mathrm{p}<0.1 * * \mathrm{p}<0.05 * * * \mathrm{p}<0.01$

(Source: conducted by author)

As shown in the table above, a 2 equipment and facilities are the first indicators of the model, which further shows that medical institutions have relatively complete and developed medical devices and facilities for elderly patients to meet the needs of diagnosis and treatment, and affect the pain feeling of patients. Specifically, A21 bed use experience and a25 rehabilitation facilities have strong significance, which is closely related to pain feeling in $99 \%$ confidence space, and the 
coefficient is negative, which shows that good ward and rehabilitation facilities environment can significantly improve the pain feeling of patients, especially the influence of bed use experience is more significant. After that, landscape maintenance entered the model, but only the impact of sports space was significant. To be significant at $99 \%$ confidence space level, it showed that the construction and maintenance of recuperation landscape in medical institutions are still insufficient, and the existing landscape maintenance and other work are also more related to sports places, and lack of the concept of environmental landscape construction for a wider recuperation place. Considering the coefficient, the influence of sports place is stronger than that of bed use experience and rehabilitation facilities, and the significant level of rehabilitation facilities also decreases, which shows that it is significant at $95 \%$ confidence space level, which indicates the positive value of sports environment maintenance and maintenance. Finally, the model introduces artificial service, whose influence is focused on rehabilitation and service attitude, the latter is more significant, which is significant at $99 \%$ confidence space level, weak in rehabilitation, significant at $95 \%$ confidence space level, and the impact of rehabilitation facilities is weakened to insignificant in this sub-level. The results show that rehabilitation activities are related to rehabilitation facilities, and show more significant effects of patients' daily life and service attitude on pain perception. Combined with the results of descriptive statistical analysis, it can be seen that the significant influence and high coefficient level of this index reflect that patients' dependence on interpersonal communication activities is relatively strong, and this kind of connection will directly affect patients' pain feelings.

\subsection{Structural Equation Model}

Finally, structural equation model analysis is carried out for the above data, and the results are as follows:

Table 6: Model regression coefficient summary

\begin{tabular}{llllllll}
\hline $\mathrm{X}$ & $\rightarrow$ & $\mathrm{Y}$ & $\begin{array}{l}\text { Nonstandard path } \\
\text { coefficient }\end{array}$ & $\mathrm{SE}$ & $\begin{array}{l}\mathrm{z}(\mathrm{CR} \\
\text { value })\end{array}$ & $\mathrm{p}$ & $\begin{array}{l}\text { Standardized path } \\
\text { coefficient }\end{array}$ \\
\hline $\mathrm{x}$ & $\rightarrow$ & $\mathrm{y}$ & 0.189 & 0.4 & 0.472 & 0.63 & 0.122 \\
1 & 1 & & 0.73 & -1.828 & 0.06 & -0.273 \\
$\mathrm{x}$ & $\rightarrow$ & $\mathrm{y}$ & -1.338 & 2 & & 8 & \\
2 & 1 & & 0.21 & -3.035 & 0.00 & -0.812 \\
$\mathrm{x}$ & $\rightarrow$ & $\mathrm{y}$ & -0.646 & 3 & 2 & \\
3 & & 1 & & & & \\
\hline
\end{tabular}

(Source: conducted by author) 
As shown in the table above, $\mathrm{x} 3$ has a significant effect on $\mathrm{y}$, and the situation of artificial service will significantly affect the chronic pain of patients, which is significant in $99 \%$ confidence space. Comparing the results of the structural equation model and hierarchical regression, it can be seen that equipment and facilities have a more significant impact on patients' direct pain, especially the bed use experience, and other indicators can significantly improve patients' pain perception. However, from the perspective of the overall research framework, the complete chronic pain should be put into the overall cognitive model, and the corresponding index analysis results show that the overall impact of artificial services on chronic pain is more significant, which cannot be effectively described by the direct feelings and experiences of patients. However, the equipment and facilities that had a significant effect on pain perception in hierarchical regression were relatively weak in the structural equation model, but still significant in the $90 \%$ confidence space. This result also shows that equipment and facilities are still kind of factors that have a significant impact on patients' chronic pain. Then, we analyze the structure of the subdivision index under each index of the structural equation model, and the other results are shown in the following table:

Table 7: Summary table of measurement expression relationship

\begin{tabular}{|c|c|c|c|c|c|c|c|}
\hline $\mathrm{X}$ & $\rightarrow$ & $Y$ & Nonstandard load factor & $\mathrm{SE}$ & $\mathrm{Z}$ & $\mathrm{p}$ & Standard load factor \\
\hline a12 & $\rightarrow$ & $\mathrm{x} 1$ & 1 & - & - & - & 0.515 \\
\hline a11 & $\rightarrow$ & $\mathrm{x} 1$ & 0.853 & 0.128 & 6.654 & 0 & 0.535 \\
\hline a13 & $\rightarrow$ & $\mathrm{x} 1$ & 0.613 & 0.112 & 5.455 & 0 & 0.404 \\
\hline a14 & $\rightarrow$ & $\mathrm{x} 1$ & 0.648 & 0.103 & 6.303 & 0 & 0.493 \\
\hline a15 & $\rightarrow$ & $\mathrm{x} 1$ & 1.821 & 0.223 & 8.17 & 0 & 0.776 \\
\hline $\mathrm{a} 22$ & $\rightarrow$ & $\mathrm{x} 2$ & 1 & - & - & - & 0.134 \\
\hline a21 & $\rightarrow$ & $\mathrm{x} 2$ & 6.352 & 3.112 & 2.041 & 0.041 & 0.814 \\
\hline $\mathrm{a} 23$ & $\rightarrow$ & $x 2$ & 3.911 & 1.949 & 2.007 & 0.045 & 0.533 \\
\hline $\mathrm{a} 24$ & $\rightarrow$ & $\mathrm{x} 2$ & 5.353 & 2.629 & 2.036 & 0.042 & 0.741 \\
\hline $\mathrm{a} 25$ & $\rightarrow$ & $x 2$ & 4.787 & 2.361 & 2.028 & 0.043 & 0.657 \\
\hline $\mathrm{a} 26$ & $\rightarrow$ & $x 2$ & 3.103 & 1.58 & 1.964 & 0.049 & 0.408 \\
\hline a32 & $\rightarrow$ & x3 & 1 & - & - & - & 0.833 \\
\hline a31 & $\rightarrow$ & $\mathrm{x} 3$ & 1.003 & 0.063 & 15.809 & 0 & 0.809 \\
\hline a33 & $\rightarrow$ & x3 & 1.017 & 0.066 & 15.456 & 0 & 0.796 \\
\hline a34 & $\rightarrow$ & x3 & 0.781 & 0.061 & 12.74 & 0 & 0.693 \\
\hline a35 & $\rightarrow$ & $\mathrm{x} 3$ & 0.895 & 0.063 & 14.232 & 0 & 0.752 \\
\hline b2 & $\rightarrow$ & y1 & 1 & - & - & - & 0.685 \\
\hline b12 & $\rightarrow$ & y1 & -1.126 & 0.096 & -11.707 & 0 & -0.798 \\
\hline b11 & $\rightarrow$ & y1 & 0.614 & 0.104 & 5.924 & 0 & 0.385 \\
\hline b13 & $\rightarrow$ & y1 & 0.441 & 0.094 & 4.686 & 0 & 0.303 \\
\hline b14 & $\rightarrow$ & $\mathrm{y} 1$ & 0.768 & 0.097 & 7.957 & 0 & 0.523 \\
\hline
\end{tabular}




\begin{tabular}{|c|c|c|c|c|c|c|c|}
\hline b15 & $\rightarrow$ & y1 & -1.204 & 0.102 & -11.815 & 0 & -0.807 \\
\hline b32 & $\rightarrow$ & $\mathrm{y} 1$ & -0.34 & 0.085 & -3.989 & 0 & -0.257 \\
\hline b31 & $\rightarrow$ & y1 & 0.138 & 0.097 & 1.42 & 0.156 & 0.091 \\
\hline b33 & $\rightarrow$ & y1 & 0.064 & 0.099 & 0.646 & 0.518 & 0.041 \\
\hline b34 & $\rightarrow$ & $\mathrm{y} 1$ & 0.103 & 0.097 & 1.057 & 0.291 & 0.068 \\
\hline b35 & $\rightarrow$ & $\mathrm{y} 1$ & 0.141 & 0.095 & 1.476 & 0.14 & 0.095 \\
\hline b42 & $\rightarrow$ & y1 & 0.097 & 0.093 & 1.039 & 0.299 & 0.067 \\
\hline b41 & $\rightarrow$ & $\mathrm{y} 1$ & -0.086 & 0.091 & -0.948 & 0.343 & -0.061 \\
\hline b43 & $\rightarrow$ & $\mathrm{y} 1$ & -0.111 & 0.067 & -1.663 & 0.096 & -0.107 \\
\hline b44 & $\rightarrow$ & $\mathrm{y} 1$ & -0.072 & 0.05 & -1.435 & 0.151 & -0.092 \\
\hline
\end{tabular}

(Source: conducted by author)

As shown in the above table, the relationship between most indicators is significant.

For independent variables, the correlation between the indicators is close. Among them, landscape maintenance and other sub-indicators have a significant impact on landscape maintenance, which is significant and positive in $99 \%$ confidence space, showing a good direction consistency. Among them, the influence of motion space is more significant, and the standardized load coefficient is higher. The significance of each index of equipment and facilities is weak, only significant in $95 \%$ confidence space, and the coefficient of each index is positive, which shows that the maintenance of equipment and facilities also has significant consistency. The impact of the three sub-indicators of human services on human services is also significant and positive in 99\% confidence space, and the coefficient of each indicator is generally high, which indicates that each item in human services generally has a strong internal connection, which may be closely related to the evaluation basis of such indicators and the corresponding service sources.

For the dependent variable, the relationship is weak and the directivity is unstable. Among them, the impact of emotional feeling is more significant, each index has a significant impact on the overall variables of emotional feeling, $\mathrm{P}$ values are all 0 , the indicators are generally significant in $99 \%$ confidence space, but the direction is not consistent. Among them, depression and fatigue are opposite to other emotions, and the absolute value of the coefficient is higher, reflecting that such emotional feelings may be associated with a better mental state or social environment. In contrast, other types of emotions have a relatively weak influence on the overall feeling of emotion, and the coefficient is relatively small, which reflects that the emotional fluctuation of patients may be more affected by more factors than chronic pain. The relationship between patients' performance and daily behavior and patients' pain feeling is weak. Chronic pain is less affected by such factors, and there is no significant relationship between indicators. Only the relationship 
between patients and their families has a weak impact on patients' chronic pain, with a P-value of 0.096, which is significant in $90 \%$ confidence space, and the coefficient is -0.107 , which is weak and negative. In other words, the relationship between the patient and his family slightly improved the patient's chronic pain.

\section{Conclusion}

Summing up the above empirical analysis results, we can see that, firstly, chronic pain is mainly affected by service attitude, secondly, it is also affected by rehabilitation and sports activities of patients. Finally, chronic pain of patients is often manifested as fear, anxiety, insomnia, unable to go out for a walk, and other symptoms. In view of such characteristics, the recuperation environment of medical institutions can be improved and improved by corresponding measures, to provide some protective measures for patients with chronic pain.

First of all, by improving the quality of nursing intervention, especially the service attitude of service staff, we can effectively improve the chronic pain symptoms of patients, help patients improve their quality of life of patients, and provide patients with a certain interpersonal environment. Secondly, the rehabilitation activities of patients are not only an important part of treatment but also a specific type of physical exercise. They can also undertake the corresponding functions of improving the social atmosphere and emotional state of patients. Therefore, the recuperation environment of medical institutions needs to provide certain physiological and psychological protection measures for patients with chronic pain by improving the sports environment and rehabilitation facilities, to improve the emotional state and motor function of patients. Finally, a series of emotional problems faced by patients, such as anxiety, fear, and insomnia, need to be paid enough attention by medical institutions, to block and protect the pain from the perspective of psychology. Due to a limited number of samples and areas being covered, the research has its limitations. Future research should cover a variety of samples and areas.

\section{REFERENCES}

An, ssLijuan. (2020). Influence of anesthesia on chronic pain and recurrence after open inguinal hernia repair and its risk factors. Unpublished doctoral dissertation. M.A. Thesis. Lanzhou: Lanzhou University. 
Cao, Chenguang, Zhongxia Liu, Bingbing Yu, Lijuan Feng. (2019). Research and Analysis on the introduction of yoga during recuperation of submarine officers and soldiers with occupational low back pain. Chinese Recuperation Medicine, 28(07), 678-680. doi: 10.13517/j.cnki.ccm.2019.07.003

Chen, Xiaohui, Xiaoqiang, Yabing, Ma, Li Ge, Zhongyuan Hu, Wenjun Yan. (2019). Research Progress on the mechanism of postoperative cognitive dysfunction in elderly patient caused by postoperative pain. Southern Medical University Press, 39(09), 1122-1126. doi: 10.12122/j.issn.1673-4254.2019.09.20

Fu, Xinying, Xuqi Shen, Xian Yang, Yun Ye, Zhiping Yu, Haiyan Bao, \& Zhijuan Li. (2020). N Application effect of lumbodorsal muscle functional exercise combined with rehabilitation physiotherapy in recuperations with lumbar disc herniation. Chinese Recuperation Medicine, 29 (05), 530-532. doi: 10.13517/j.cnki.ccm.2020.05.030

Gao, Meihua. (2019). Influencing factors and protective measures of chronic pain of elderly recuperations in a sanatorium. Nursing Practice and Research, 16(17), 60-61. doi: 10.3969/j.issn.1672-9676.2019.17.027.

Han, Jian, Dongyue Li, Dan Lv, Yongjin He. (2020). Research status of self-management of chronic pain in the elderly. China's Urban and Rural Enterprise Health, 35 (07), 93-95. doi: 10.16286/j.1003-5052.2020.07.033

Jiang, Yanfang, Qian Zhang, Muzi Li, Tong Wang, \& Tao Wu. (2020). Effect of acupuncture combined with massage on pain improvement and lumbar flexion activity in patients with neck, shoulder, waist, and leg pain. Chinese Recuperation Medicine, 29 (08), 840-841. doi: 10.13517/j.cnki.ccm.2020.08.020

Li, Xiahui, Jiping Li, Fan Yang. (2020). Research progress of chronic pain education for the elderly. Shanghai Nursing, 20 (12), 49-52. doi: 10.3969/j.issn.1009-8399.2020.12.012

Lu, Yi, Jiayin Yao, Chulian Gong, Bao Wang, Zhaohui Qin, Junming Lao, Dong Liu, Xinhua Yao. (2019). Related factors and prediction model of chronic postoperative pain in elderly patients undergoing hip replacement. Journal of Clinical Anesthesiology, 35 (12), 1197-1200. doi: CNKI:SUN:LCMZ.0. 2019-12-015

Pei, Jinxue, Yue Long, \& Wei Wei. (2020). Effect of individualized hot spring recuperation combined with physical therapy on rehabilitation of patients with chronic low back and 
leg pain. Chinese Recuperation Medicine, 29 (08), 828-829. doi:

10.13517/j.cnki.ccm.2020.08.015

Rajput, Hira Islam,Muhammad Atif Khan,Muhammad Asif,Muzna Kafeel,Khadija Tul

Kubra,Muhammad Jawed Baig Chughtai,\& Muhammad Riaz Baig Chughtai.(2019).

Prevalence of work-related neck and shoulder pain among office receptionists of Karachi. Journal of Contemporary Medicine, 19 (2), 123-127. https://doi.org/10.16899/jcm.571620

Shen, Juping. (2019). Experience of clinical application value of rehabilitation physiotherapy in recuperations with lumbar disc herniation. World's Latest Medical Information Digest, 19 (11), 40. doi: 10.19613/j.cnki.1671-3141.2019.11.024

Shen, Yao, Mengxia Zhao, Linglin Kong, Xiao Liu, Fanying Li, Yuchang Song, Ling Wu, Tie Zou, Xinlian Yang, Qianlong Zhang. (2019). Investigation and analysis of pain status of elderly patients with chronic pain in Xianning community. Journal of Hubei University of Science and Technology (Medical Edition), 33 (02), 158-161. doi: 10.16751/j.cnki.20954646.2019.02.0158

Song, Yuchang, Mengxia Zhao, Linglin Kong, Fanying Li, Yao Shen, Ling Wu. Yi Zou. (2019). Investigation on quality of life of elderly patients with chronic pain in the community. Medical Information, 32 (02), 135-138. https://doi.org/10.1055/s-0038-1676480

Wang, Jianhua, Xiuling Zhong, Feng Li, Liling Su \& Kailan Song (2019). Research progress of the quality of life in elderly patients with chronic pain. Occupation and Health, 35 (21), 3003-3006. doi:CNKI:SUN:ZYJK.0.2019-21-030

Wang, Ting, Yun Liu. (2019). Current situation of chronic pain in the elderly and progress of nursing intervention [J]. Modern Medicine and Health, 35 (01), 79-81. doi:

CNKI:SUN:XYWS.0.2019-01-022

Wang, Xinjing, Xiaohong Yu, Feng Huang, Xianzhong Luo \& Yan Gong (2020) Effect of acupuncture combined with fire acupuncture on pain and serum inflammatory factors in patients with lumbar disc herniation. Progress in Modern Biomedicine, 20 (17), 33093312. doi: 10.13241/j.cnki.pmb.2020.17.024

$\mathrm{Xu}$, Lihua, Ruihua Jing, \&Wanying Liu. Application of emotional release therapy in elderly patients with chronic pain in nursing homes. Modern Clinical Nursing, 19 (07), 55-62. doi:10.3969/j.issn.1671-8283.2020.07.009 
Yan, Fei. (2019). Research progress of chronic pain management in elderly patients. Forum on Primary Medicine, 23 (12), 1750-1751. doi: 10.19435/j.1672-1721.2019.12.085

Zhang, Li, Yumei Li, Muqing He \& Hong Sun. (2021). Application effect of community highquality nursing based on intervention quality control group in elderly patients with chronic pain. International Journal of Nursing, 40 (03), 566-588. doi: 10.3760/cma.j.cn221370-20190108-00167

Zhao, Wenwen, Zhaowen Peng, Jing Yang. (2019). Evaluation of the effect of holistic pain assessment scale in elderly patients with chronic pain. Journal of contemporary clinical medicine, 32 (03), 242-202. doi: 10.3969/j.issn.2095-9559.2019.03.026

Zhu, Chengwei (2020) Quality of life of elderly patients with chronic pain in the community. Community Physicians in China, 36 (26), 181-182. doi: https://doi.org/10.1016/S1530$\underline{891 \times(20) 39485-4}$ 